\title{
A Novel Dead-Time Vector Approach to Analysis of DC Link Current in PWM Inverter Drives
}

\author{
C.C. Chan, K.T. Chau, and Y. Li \\ Department of Electrical \& Electronic Engineering, \\ The University of Hong Kong, Pokfulam Road, Hong Kong
}

\begin{abstract}
An analysis on the DC link current of spacevector PWM drives using novel dead-time vector (DTV) concept has been presented. By employing the DTV, a bi-value function to quantify the polarities of three phase winding currents, inverter output voltages and DC link current at dead time state can be expressed in close vectored forms. Thus, the entire drive system is considered as a whole to investigate the characteristics of DC link current at both normal and dead time states during steady and transient operations. Increasingly, a phenomenon of negative spikes on DC link current caused by dead time effect is newly observed and highlighted. This phenomenon can be accurately predicted and mathematically characterized by using the proposed DTV approach. Both computer simulation and experimental results have been used for verification.
\end{abstract}

\section{INTRODUCTION}

The knowledge of DC link current in PWM inverter-fed $\mathrm{AC}$ motor drives has been of utmost importance for system protection, efficiency optimization and state identification [1]-[3]. The available approaches, however, are unnecessarily complicated and impractical for the modern drive systems using space vector PWM schemes [4]. Even so, these approaches have been developed mainly for steady state analysis, while the characteristics of DC link current during transient operation, which are of much significance, have seldom been analyzed.

On the other hand, it is well known that "dead time" is inevitably equipped to protect inverter devices from shortthrough the power source in an inverter. Since dead time has great influence on the performance of drive systems [5], [6], it is urgently need to know the precise information of dead time in a practical drive system. Because of the time delay in the gate driver circuit, however, the duration of dead time in the main circuit is not the same as that set in the control board, especially at high power rating. And it is difficult to directly measure dead time at the main power modules when the drive system is operating. Fortunately, the DC link current can easily be non-invasively measured. It has been observed that there are both positive and negative spikes on the instantaneous DC link current waveform. It is apt to consider these spikes as the switching transients of power devices, such as reverse recovery effect of freewheeling diodes, and omit them as EMI issue. But, are all the current spikes exclusively caused by the switching transients? Does the existence of dead time also cause any spikes on the instantaneous DC link current waveforms? Or can the information of dead time be deduced from DC link current? And how? To the knowledge of authors, the available studies on dead time effect are mainly focus on the inverter output side and the analysis of dead time effect on DC link current is poorly inadequate.

In this paper, a comprehensive analysis on DC link current of space vector PWM induction motor drives in the presence of dead time is presented. With the introduction of a novel concept - dead time vector (DTV), which is a bi-value function to quantify the polarities of three-phase winding currents, the inverter output voltages and DC link current at dead time can be expressed in close vectored forms. Thus, the entire drive system, including PWM strategy, inverter and motor, is considered as a whole to investigate the characteristics of DC link current at both normal and dead time states during steady and transient operations. Moreover, the phenomenon of spikes caused by dead time effect on the DC link current is not only descriptively discussed and explained, but also mathematically predicted and derived using the proposed DTV approach. Theoretical analysis and computer simulation are verified by experimental results.

\section{UNIFIED APPROACH}

A voltage source inverter-induction motor drive system is shown in Fig. 1, where $I_{d c}$ is the instantaneous DC link current, $I_{u}, I_{v}$ and $I_{w}$ are the instantaneous three phase upper leg currents, $I_{a}, I_{b}$ and $I_{c}$ are the instantaneous three phase winding currents. And the nominal current directions are indicated as the arrow signs.

Generally, the current flows inside the inverter are affected by the diode recovery and snubber action. When a switch is being turned-on and the conducting diode at the same leg is being blocked off by this turn-on, because of the reverse recovery effect of diode, this leg is in fact shorted through at this moment such that a positive current spike will appear at the DC link side. And the snubbers are for strayinductance clamping and turn-off switching loss reduction. The main concern here is to establish the relationships between DC link current, windings currents and device switching patters. So it is assumed that the inverter switches are "ideal": both on-state losses and switching times are negligible, the diode recovery effect and snubber action are not considered either. 


\section{A. Space Vectors for Normal States}

During normal states, there are eight switching states, which can be expressed as the space voltage vector $\operatorname{SVV}\left(S_{a}, S_{b}, S_{c}\right)$, such as $(0,0,0),(0,0,1),(0,1,0), \ldots,(1,1,1)$, where $S_{p}=0 \quad(p=a, b, c)$ means that the upper leg switch of phase $p$ is off while the lower one is on, and vice versa. The three phase winding terminal voltage potentials are

$\left\{\begin{array}{l}U_{a}=\frac{1}{2} U_{d c}\left(2 S_{a}-1\right) \\ U_{b}=\frac{1}{2} U_{d c}\left(2 S_{b}-1\right) \\ U_{c}=\frac{1}{2} U_{d c}\left(2 S_{c}-1\right)\end{array}\right.$

The switching vectors describe the inverter output voltages as shown in Fig. 2. Projecting voltage vectors onto a two-dimension $\alpha, \beta$ plane, the $\alpha, \beta$ components can be derived as:

$\left[\begin{array}{l}U_{\alpha} \\ U_{\beta}\end{array}\right]=\sqrt{\frac{2}{3}}\left[\begin{array}{ccc}1 & -\frac{1}{2} & -\frac{1}{2} \\ 0 & \frac{\sqrt{3}}{2} & -\frac{\sqrt{3}}{2}\end{array}\right]\left[\begin{array}{l}S_{a} \\ S_{b} \\ S_{c}\end{array}\right] U_{d c}$

Once the voltage vectors are derived and the load property is known from mathematical model of the motor, the winding currents can be calculated out.

The motor winding currents flow via either the positive or negative supply rail depending on the switching states. Although under different cases, the conducting devices may be different, their effects are the same - for instance, current flowing in switch $S_{1}$ or freewheeling diode $D_{1}$ will result in the winding terminal point $A$ being connected to the positive supply rail.

Phase A is considered first. When $S_{a}=1$, the winding terminal point $\mathrm{A}$ is connected to the positive supply rail, and the current flows through the upper leg of the inverter $\left(S_{1}\right.$ conducts if $I_{a}>0$ and $D_{1}$ conducts if $\left.I_{a}<0\right)$, thus $I_{u}=I_{a}$; when $S_{a}=0$, the winding terminal point A is connected to the negative supply rail, and the current flows through the lower leg of the inverter $\left(D_{4}\right.$ conducts if $I_{a}>0$ and $S_{4}$ conducts if $I_{a}<0$ ), there is no current flowing through the upper leg of the inverter, thus $I_{u}=0$. The situations for phase $B$ and $C$ are similar.

From the analysis above, the relation between leg currents and switching states are derived as

$$
\left\{\begin{array}{l}
I_{u}=I_{a} S_{a} \\
I_{v}=I_{b} S_{b} \\
I_{w}=I_{c} S_{c}
\end{array}\right.
$$

As the instantaneous DC link current is the sum of inverter three leg currents, that is

$I_{d c}=I_{u}+I_{v}+I_{w}$

Substituting (3) into (4) yields
$I_{d c}=I_{a} S_{a}+I_{b} S_{b}+I_{c} S_{c}$

Equation (5) is the relation between $I_{d c}$ and three phase winding currents derived from the view of switching state transitions. Actually, it can also be derived from the view of power flow inside the inverter. A deeper insight on inverter operation will be exposed by means of the derivation.

Inverter output instantaneous three phase power is always equal to $\mathrm{DC}$ input power to the inverter, thus

$U_{d c} I_{d c}=U_{a n} I_{a}+U_{b n} I_{b}+U_{c n} I_{c}$

where $U_{a n}, U_{b n}$ and $U_{c n}$ are the three phase voltages of motor windings respectively. Let $U_{n}$ be the voltage potential of the neutral point $\mathrm{N}$, considering the terminal voltage potentials as in (1), we get

$$
U_{a n} I_{a}+U_{b n} I_{b}+U_{c n} I_{c}
$$

$=\left(U_{a}-U_{n}\right) I_{a}+\left(U_{b}-U_{n}\right) I_{b}+\left(U_{c}-U_{n}\right) I_{c}$

$=U_{d c}\left(S_{a} I_{a}+S_{b} I_{b}+S_{c} I_{c}\right)-\left(\frac{1}{2} U_{d c}+U_{n}\right)\left(I_{a}+I_{b}+I_{c}\right)$

Because of the constraint of $I_{a}+I_{b}+I_{c}=0$, by comparing (7) with (6), the DC link current is again derived as $I_{d c}=I_{a} S_{a}+I_{b} S_{b}+I_{c} S_{c}$

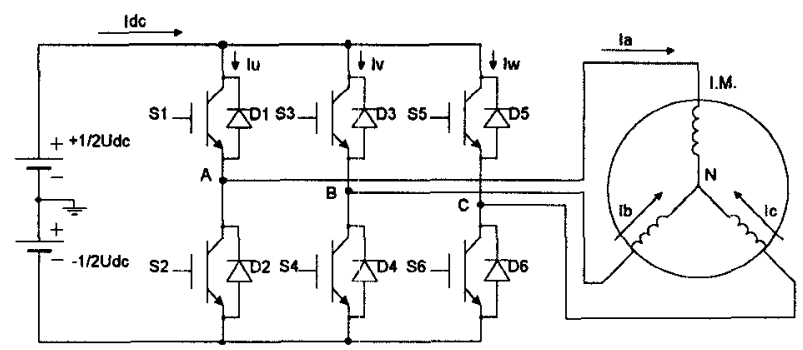

Fig. 1. Three-phase PWM inverter with an induction motor load.

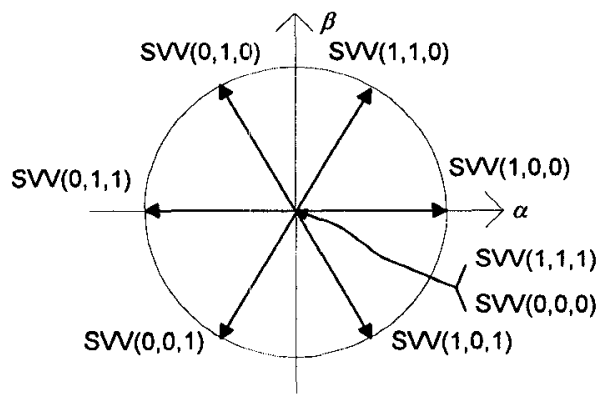

Fig. 2. Voltage vectors and space sectors.

\section{B. Dead-Time Vectors}

During dead time period, both two series-connected switches on the same leg are Off and the winding currents are flowing through the freewheeling diodes, as determined by the polarities of winding currents but independent of the switching states - upper diodes if currents flow back to supply and lower diodes if currents flow forward to load. 
Dead time state of phase A is illustrated as Fig. 3. If the switching state of phase $\mathrm{A}$ is scheduled to be changed, i.e. from the state of $S_{1}$ On and $S_{2}$ Off to the state of $S_{1}$ Off and $S_{2}$ On or vice versa, a dead time is inserted before the scheduled switching action happens. At this interval, both $S_{1}$ and $S_{2}$ are Off and there are two modes for the current flow.

\section{- Mode 1: $I_{a}>0$.}

The current flows forward to load through the diode $D_{2}$ at the lower leg of the inverter. Terminal point $A$ is connected to the negative supply rail, and there is no current flowing through the upper leg. Thus the inverter leg current and terminal potential are

$I_{u}=0$

$$
\begin{aligned}
U_{a} & =\left(-\frac{1}{2}\right) U_{d c} \\
& =\frac{1}{2} U_{d c}(0-1)
\end{aligned}
$$

- Mode 2: $I_{a}<0$.

The current flows back to the supply through the diode $D_{1}$ at the upper leg of the inverter. Terminal point $A$ is connected to the positive supply rail, and there is no current flowing through the lower leg. Thus the inverter leg current and terminal potential are

$$
\begin{aligned}
I_{u} & =I_{a} \\
U_{a} & =\left(+\frac{1}{2}\right) U_{d c} \\
& =\frac{1}{2} U_{d c}(2-1)
\end{aligned}
$$

Obviously, during dead time interval, the currents and voltages greatly depend on the polarities of winding currents, which are often expressed as a function defined as [6]

$\operatorname{Sign}\left(I_{p}\right)=\left\{\begin{array}{ccc}1 & \text { when } & I_{p}>0 \\ -1 & \text { when } & I_{p}<0\end{array}\right.$ where $p=a, b, c$

However, it is inconvenient to directly use Sign $\left(I_{p}\right)$ to express the currents and voltages at dead time intervals into simple and clear forms. Thus a novel concept - dead time vector is proposed. For three phases $A, B$ and $C$, $\operatorname{DTV}\left(D_{a}, D_{b}, D_{c}\right)$ is defined as

$D_{p}=\frac{1}{2}\left[1-\operatorname{Sign}\left(I_{p}\right)\right] \quad$ where $p=a, b, c$

Under this definition, the physical meaning of $D T V$ is

$D_{p}=\left\{\begin{array}{lll}0 & \text { when } & I_{p}>0 \\ 1 & \text { when } & I_{p}<0\end{array}\right.$ where $p=a, b, c$

Thus $D T V$ is a bi-value $[0,1]$ function similar to $S V V$ in form. By introducing $D T V$ to (9) - (12), the inverter upper leg current and terminal potential are derived from the analysis above as

$I_{u}=I_{a} D_{a}$

$U_{a}=\frac{1}{2} U_{d c}\left(2 D_{a}-1\right)$
Similarly, if phase B and phase $\mathrm{C}$ are at dead time state respectively (not necessarily simultaneous), we have

$I_{v}=I_{b} D_{b}$

$U_{b}=\frac{1}{2} U_{d c}\left(2 D_{b}-1\right)$

and

$I_{w}=I_{c} D_{c}$
$U_{c}=\frac{1}{2} U_{d c}\left(2 D_{c}-1\right)$

Totally, there are $3^{3}-8=19$ combinations of dead time states for three phase inverters which can be categorized into three cases to express output voltages and DC link currents at dead time interval:

- Case 1: change in switching state of only one phase (phase A changes while both $\mathrm{B}$ and $\mathrm{C}$ remain unchanged, thus phase $\mathrm{A}$ is at dead time state and phase $\mathrm{B}, \mathrm{C}$ are at normal switching states).

The three phase terminal voltage potentials are

$\left\{\begin{array}{l}U_{a}=\frac{1}{2} U_{d c}\left(2 D_{a}-1\right) \\ U_{b}=\frac{1}{2} U_{d c}\left(2 S_{b}-1\right) \\ U_{c}=\frac{1}{2} U_{d c}\left(2 S_{c}-1\right)\end{array}\right.$

The output voltage vector is

$\left[\begin{array}{l}U_{\alpha} \\ U_{\beta}\end{array}\right]=\sqrt{\frac{2}{3}}\left[\begin{array}{rrr}1 & -\frac{1}{2} & -\frac{1}{2} \\ 0 & \frac{\sqrt{3}}{2} & -\frac{\sqrt{3}}{2}\end{array}\right]\left[\begin{array}{l}D_{a} \\ S_{b} \\ S_{c}\end{array}\right] U_{d c}$

The instantaneous DC link current is

$$
\begin{aligned}
I_{d c} & =I_{u}+I_{v}+I_{w} \\
& =I_{a} D_{a}+I_{b} S_{b}+I_{c} S_{c}
\end{aligned}
$$
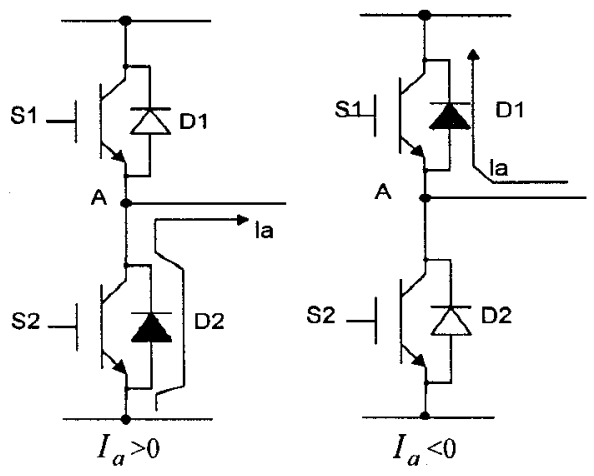

Fig. 3. Current flow at dead time interval for phase A

- Case 2: change in switching states of two phases (both phase $A$ and $B$ change while $C$ remains unchanged, thus phase $A$ and $B$ are at dead time states and phase $C$ is at normal state). 
The three phase terminal voltage potentials are

$\left\{\begin{array}{l}U_{a}=\frac{1}{2} U_{d c}\left(2 D_{a}-1\right) \\ U_{b}=\frac{1}{2} U_{d c}\left(2 D_{b}-1\right) \\ U_{c}=\frac{1}{2} U_{d c}\left(2 S_{c}-1\right)\end{array}\right.$

The output voltage vector is

$\left[\begin{array}{l}U_{\alpha} \\ U_{\beta}\end{array}\right]=\sqrt{\frac{2}{3}}\left[\begin{array}{rrr}1 & -\frac{1}{2} & -\frac{1}{2} \\ 0 & \frac{\sqrt{3}}{2} & -\frac{\sqrt{3}}{2}\end{array}\right]\left[\begin{array}{l}D_{a} \\ D_{b} \\ S_{c}\end{array}\right] U_{d c}$

The instantaneous DC link current is

$I_{d c}=I_{u}+I_{v}+I_{w}$

$=I_{a} D_{a}+I_{b} D_{b}+I_{c} S_{c}$

- Case 3: change in switching states of all phases (but usually absent in normal switching schemes for induction motor drive so not be discussed here).

Therefore, thanks to the introduction of $D T V$, the voltage vectors (23), (26) and instantaneous DC link currents (24), (27) for dead time intervals are expressed in the similar form of their counterparts (1) and (8) for normal switching intervals which are derived from $S V V$. So the inverter, no matter operating at either normal or dead time interval, can be analyzed in a unified form as a whole instead of looking at the individual phase separately. It is convenient and useful for performance prediction, digital simulation and controller design. However, it should be addressed that although DTV has the similar form with $S V V$, they inherently are different in physical meanings. DTV reflects the polarities or directions of winding currents while $S V V$ reflects the switching states of the inverter.

\section{FORMATION OF NEGATIVE SPIKES}

In this section, the newly proposed DTV and expressions for DC link currents will be used to discuss the question raised at Introduction section, that is, whether the existence of dead time causes some spikes at DC link current and how. Here current spike means that the DC link current at dead time interval is much smaller or larger than those at its adjacent normal switching subcycles. Since dead time duration is much shorter than switching period, the current spikes are seen on DC link current waveforms.

The transition of switching states at two adjacent subcycles is illustrated by Fig. 4. In subcycle $T_{n}$, the applied voltage vector is $S V V_{n}$; in the next subcycle $T_{n+1}$, the applied voltage vector is $S V V_{n+1}$. Between $T_{n}$ and $T_{n+1}$, dead time $T_{d}$ is inserted. For the reason of simplicity and clarity, it is assumed that winding currents $I_{a}, I_{b}$ and $I_{c}$ keep unchanged at dead time interval because of winding inductance and high switching frequency.

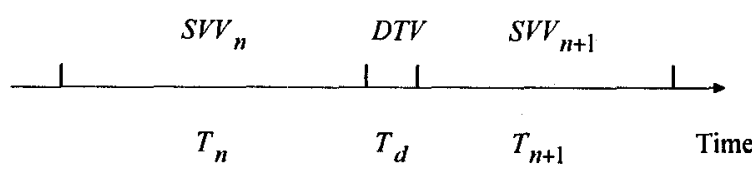

Fig. 4. Transition of switching states.

General space vector PWM switching schemes for induction motor control ensure that the case for three phase switching states changing simultaneously does not happen, so there are two cases for the change of $I_{d c}$ at dead time interval which are analyzed as follows:

- Case 1: change in switching state of only one phase (phase A change while phase B, C remain unchanged).

Voltage vector changes from $S V V_{n}\left(S_{a, n}, S_{b, n}, S_{c, n}\right)$ to $S V V_{n+1}\left(S_{a, n+1}, S_{b, n}, S_{c, n}\right)$.

Before $T_{d}, I_{d c, n}=I_{a} S_{a, n}+I_{b} S_{b, n}+I_{c} S_{c}$

During $T_{d}, I_{d c, \text { dead }}=I_{a} D_{a, n}+I_{b} S_{b, n}+I_{c} S_{c}$

After $T_{d}, I_{d c, n+1}=I_{a} S_{a, n+1}+I_{b} S_{b, n}+I_{c} S_{c}$

Since both $D T V$ and $S V V$ are bi-value $[0,1]$ functions, obviously there are only two results

$$
\begin{array}{ll}
I_{d c, \text { dead }}=I_{d c, n} & \text { when } D_{a}=S_{a, n} \\
I_{d c, \text { dead }}=I_{d c, n+1} & \text { when } D_{a}=S_{a, n+1}
\end{array}
$$

As $I_{d c}$ at dead time interval is equal to that at either one of its adjacent normal switching subcycles, there is definitely no spikes on DC link current caused by dead time at case 1 .

- Case 2: change in switching states of two phases (phases $A$ and $B$ change while phase $C$ remain unchanged).

Voltage vector changes from $S V V_{n}\left(S_{a, n}, S_{b, n}, S_{c, n}\right)$ to $S V V_{n+1}\left(S_{a, n+1}, S_{h, n+1}, S_{c, n}\right)$.

Before $T_{d}, I_{d c, n}=I_{a} S_{a, n}+I_{b} S_{b, n}+I_{c} S_{c}$

During $T_{d}, I_{d c, \text { dead }}=I_{a} D_{a}+I_{b} D_{b}+I_{c} S_{c}$

After $T_{d}, I_{d c, n+1}=I_{a} S_{a, n+1}+I_{b} S_{b, n+1}+I_{c} S_{c}$

First of all, $I_{d c, d e a d}$ is compared with $I_{d c, n}$ and $I_{d c, n+1}$. Obviously it is only needed to compare $\left(I_{a} D_{a}+I_{b} D_{b}\right)$ with $\left(I_{a} S_{a, n}+I_{b} S_{b, n}\right)$ and $\left(I_{a} S_{a, n+1}+I_{b} S_{h, n+1}\right)$ respectively.

When $\left[\begin{array}{ll}D_{a} & D_{b}\end{array}\right]=\left[\begin{array}{ll}S_{a, n} & S_{b, n}\end{array}\right], \quad I_{d c, \text { dead }}=I_{d c, n} ; \quad$ when $\left[\begin{array}{ll}D_{a} & D_{b}\end{array}\right]=\left[\begin{array}{ll}S_{a, n+1} & S_{b, n+1}\end{array}\right], I_{d c, \text { dead }}=I_{d c, n+1}$, hence there is definitely no spikes on DC link current caused by dead time.

However, when $\left[\begin{array}{ll}D_{a} & D_{b}\end{array}\right] \neq\left[\begin{array}{ll}S_{a, n} & S_{b, n}\end{array}\right]$ and $\left[\begin{array}{ll}D_{a} & D_{b}\end{array}\right] \neq\left[\begin{array}{ll}S_{a, n+1} & S_{b, n+1}\end{array}\right]$, things will be different. For example, if $\left[\begin{array}{ll}D_{a} & D_{b}\end{array}\right]=\left[\begin{array}{ll}0 & 0\end{array}\right], \quad\left[\begin{array}{ll}S_{a, n} & S_{b, n}\end{array}\right]=\left[\begin{array}{ll}0 & 1\end{array}\right]$, $\left[\begin{array}{ll}S_{a, n+1} & S_{b, n+1}\end{array}\right]=\left[\begin{array}{ll}1 & 0\end{array}\right]$, according to the physical meaning of DTV as (15), we obtain $I_{a}>0, I_{b}>0$. Since

$I_{a} S_{a, n}+I_{b} S_{b, n}=I_{b}>0$

$I_{a} S_{a, n+1}+I_{b} S_{b, n+1}=I_{a}>0$

$I_{a} D_{a}+I_{b} D_{b}=0$ 
we obtain $I_{d c, \text { dead }}<I_{d c, n}$ and $I_{d c \text {,dead }}<I_{d c, n+1}$.

There are totally eight combinations of $D T V$ and $S V V$ for this situation $\quad\left(\left[\begin{array}{ll}D_{a} & D_{b}\end{array}\right] \neq\left[\begin{array}{ll}S_{a, n} & S_{b, n}\end{array}\right] \quad\right.$ and $\left.\left[\begin{array}{ll}D_{a} & D_{b}\end{array}\right] \neq\left[\begin{array}{ll}S_{a, n+1} & S_{b, n+1}\end{array}\right]\right)$, the comparison between $I_{d c, \text { dead }}$ and $I_{d c, n}, I_{d c, n+1}$ is analyzed and presented in the same method above in Table I. Comparisons for the eight combinations all lead to $I_{d c, \text { dead }}<I_{d c, n}$ and $I_{d c, \text { dead }}<I_{d c, n+1}$.

Secondly, the value of $I_{d c \text {,dead }}$ itself is checked also, which can be calculated by (32). There are totally eight combinations of $D_{a}, D_{b}$ and $S_{c, n}$. Referring to the physical meaning of DTV and the constraint of $I_{a}+I_{b}+I_{c}=0$, $I_{d c, \text { dead }}$ are calculated and listed in Table II. It is shown that under all of the eight combinations, no matter the polarities of winding currents $I_{a}, I_{b}$ and $I_{c}$ themselves might be, we always get $I_{d c, \text { dead }} \leq 0$. Therefore, under the condition of $\left[\begin{array}{ll}D_{a} & D_{b}\end{array}\right] \neq\left[\begin{array}{ll}S_{a, n} & S_{b, n}\end{array}\right]$ and $\left[\begin{array}{ll}D_{a} & D_{b}\end{array}\right] \neq\left[\begin{array}{ll}S_{a, n+1} & S_{b, n+1}\end{array}\right]$, DC link current at dead time interval is definitely smaller than those at its adjacent normal switching subcycles and the value itself of DC link current at dead time interval is negative or zero. Since dead time duration is much shorter than switching period, there are negative current spikes occurring on DC link current waveforms.

From the discussion above, it can be concluded that if and only if two phase switching states change simultaneously, say, $S V V$ changing from $(1,0,0)$ to $(0,1,0)$, negative spikes may be appear on DC link current at dead time interval, and the inverter operation also determine the occurrence of negative spikes. The criterion for the formation of negative spikes is presented precisely and clearly by the introduction of $D T V$. Increasingly dead time only causes negative current spikes and their amplitudes are equal to either one of three phase winding currents (see Table II). These negative spikes inherently represent the regenerative operating mode at dead time interval and will significantly influence the overall system stability.

TABLE I

\begin{tabular}{|c|c|c|c|c|c|c|c|c|}
\hline $\begin{array}{l}s_{a, n} \\
\left.s_{b, n}\right]\end{array}$ & $\begin{array}{l}{\left[s_{a, n+1}\right.} \\
\left.s_{b, n+1}\right]\end{array}$ & $\begin{array}{l}{\left[D_{a}\right.} \\
\left.D_{b}\right]\end{array}$ & $I_{a}$ & $I_{b}$ & $\begin{array}{c}I_{a} S_{a, n} \\
+ \\
I_{b} S_{b, n}\end{array}$ & $\begin{array}{c}I_{a} S_{a, n+} \\
+ \\
I_{b} S_{b, n+1}\end{array}$ & $\begin{array}{c}I_{a} D_{a} \\
+ \\
I_{a} D_{b}\end{array}$ & OK? \\
\hline$[0,0]$ & {$[1,1]$} & {$[0,1]$} & $>0$ & $<0$ & 0 & $I_{a}+I_{b}$ & $I_{b}$ & $\bar{Y}$ \\
\hline$[0,0]$ & {$[1,1]$} & {$[1,0]$} & $<0$ & $>0$ & 0 & $I_{a}+I_{b}$ & $I_{a}$ & $\mathrm{Y}$ \\
\hline$[1,1]$ & {$[0,0]$} & {$[0,1]$} & $>0$ & $<0$ & $I_{a}+I_{b}$ & 0 & $I_{b}$ & $\mathrm{Y}$ \\
\hline$[1,1]$ & {$[0,0]$} & {$[1,0]$} & $<0$ & $>0$ & $I_{a}+I_{b}$ & 0 & $I_{a}$ & $\mathrm{Y}$ \\
\hline$[0,1]$ & {$[1,0]$} & {$[1,1]$} & $<0$ & $<0$ & $I_{b}$ & $I_{a}$ & $I_{a}+I_{b}$ & $\mathrm{Y}$ \\
\hline$[0,1]$ & {$[1,0]$} & {$[0,0]$} & $>0$ & $>0$ & $I_{h}$ & $I_{a}$ & 0 & $\mathrm{Y}$ \\
\hline$[1,0]$ & {$[0,1]$} & {$[1,1]$} & $<0$ & $<0$ & $I_{a}$ & $I_{b}$ & $I_{a}+I_{b}$ & $\mathrm{Y}$ \\
\hline$[1,0]$ & {$[0,1]$} & {$[0,0]$} & $>0$ & $>0$ & $I_{a}$ & $I_{b}$ & 0 & $\mathrm{Y}$ \\
\hline
\end{tabular}

TABLE II

\begin{tabular}{lllllll}
\multicolumn{6}{c}{$D_{a}, D_{b}$ and $S_{c, n}$ SATISFYING $I_{d c, \text { dead }} \leq 0$} \\
\hline$S_{c, n}$ & $D_{a}$ & $D_{b}$ & $I_{a}$ & $I_{b}$ & $I_{d c, \text { dead }}$ & $I_{d c, \text { dead }}<0 ?$ \\
\hline 0 & 0 & 0 & $>0$ & $>0$ & 0 & \\
0 & 0 & 1 & $>0$ & $<0$ & $I_{b}$ & $\mathrm{Y}$ \\
0 & 1 & 0 & $<0$ & $>0$ & $I_{a}$ & $\mathrm{Y}$ \\
0 & 1 & 1 & $<0$ & $<0$ & $I_{a}+I_{b}$ & $\mathrm{Y}$ \\
1 & 0 & 0 & $>0$ & $>0$ & $-\left(I_{a}+I_{b}\right)$ & $\mathrm{Y}$ \\
1 & 0 & 1 & $>0$ & $<0$ & $-I_{a}$ & $\mathrm{Y}$ \\
1 & 1 & 0 & $<0$ & $>0$ & $-I_{b}$ & $\mathrm{Y}$ \\
1 & 1 & 1 & $<0$ & $<0$ & 0 & \\
\hline
\end{tabular}

\section{RESULTS AND DISCUSSION}

Digital simulation on the whole inverter-induction motor drive system in the presence of dead time has been made and verified with experimental results. The flux-control space vector PWM scheme is used as in [7] and all the parameters and variables are presented in per unit (see Appendix).

\section{A. Simulation Waveforms}

The waveforms of $I_{d c}$ and $I_{a}$ at $50 \mathrm{~Hz}$ for one fundamental operation period $(20 \mathrm{~ms})$ are shown as Fig. 5 . At one operation period, there are six pulses in the waveform of $I_{d c}$. It is because that the whole $360^{\circ}$ plane is divided into six sectors by the voltage vectors as shown in Fig. 2, each spanning $60^{\circ}$. There are two selective voltage vectors as one group for each sector and the control strategy makes the inverter switching actions alike at each sector. Seen from DC link side, the waveforms at different sectors are similar.

The waveforms of instantaneous $I_{a}$ and $I_{d c}$ when the inverter-fed induction motor is being started-up from standstill at $50 \mathrm{~Hz}$ are shown in Fig. 6. There are both positive and negative values at the instantaneous DC link current during transient process because of the relatively low and changing power factor of the induction motor when it is being started-up from standstill.

Fig. 7 is the waveform of average DC link current during start-up process, which is responsible for real power transfer. It can be found that the average DC link current is very close to the output torque $T_{e}$ of the induction motor during start-up process. The reasons are due to the fact that the real power transferred from supply to induction motor is $P_{\text {real }}=U_{d c} I_{d c, \text { avg }}$, where $U_{d c}$ is the DC supply voltage and kept constant; the motor output power across air gap is $P_{\text {gap }}=T_{e} \omega_{e}$, where $\omega_{e}$ is the synchronous speed and is always equal to unity p.u.; and the stator copper and core losses are relatively small and usually changing during the start-up process. 


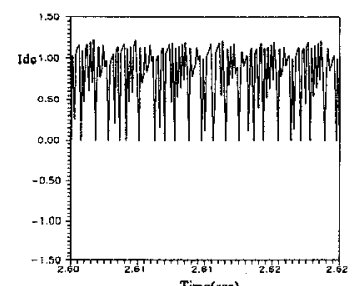

(a)

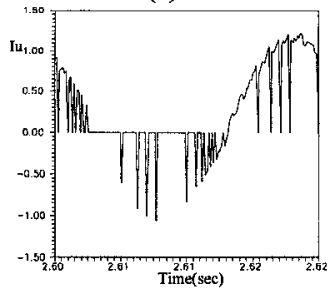

(c)

Fig. 5. Steady state waveforms without considering dead time. (a) DC link current. (b) Winding current. (c) Leg current.

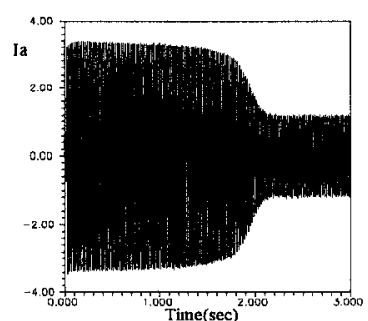

(a)

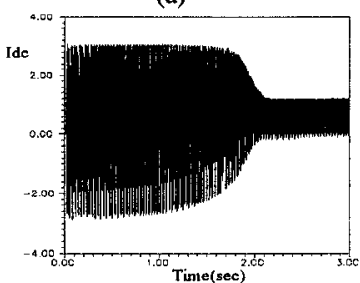

(c)

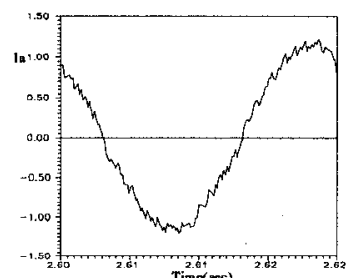

(b)

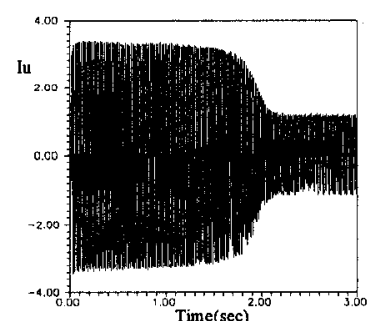

(b)

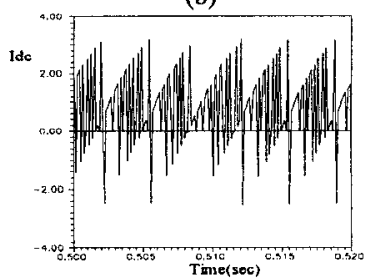

(d)
Fig. 6. Start-up transient waveforms without considering dead time. (a) Winding current. (b) Leg current. (c) DC link current. (d) DC link current zoomed to $20 \mathrm{~ms}$.

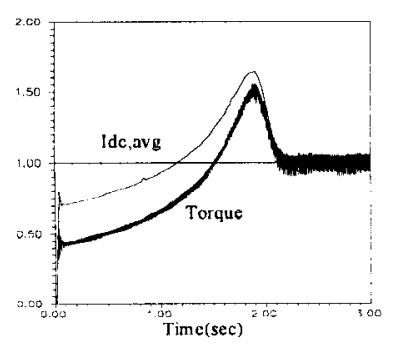

Fig. 7. Start-up average DC link current and output torque.

To illustrate the effect of dead time, waveforms of $I_{d c}$ at one fundamental operation period during steady state are shown at Fig. 8, with and without dead time being considered. If dead time is not considered, there is definitely no negative spike at $I_{d c}$. However, if dead time is considered as $T_{d}=10 \mu \mathrm{s}$, significant negative spikes are found at $I_{d c}$. The time-zoomed waveforms of the same $I_{d c}$ are shown in Fig. 8 (c) and (d) so that the negative current spikes can be seen clearly. It is found that the time width of these negative spikes is equal to $10 \mu \mathrm{s}$, which is the dead time duration. Also it is noticed that not in every subcycle there exists this kind of negative spikes. From the above figures, it can be concluded that the negative spikes on the DC link current are caused by dead time effect and influenced by inverter operation.

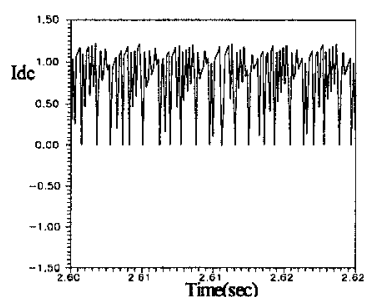

(a)

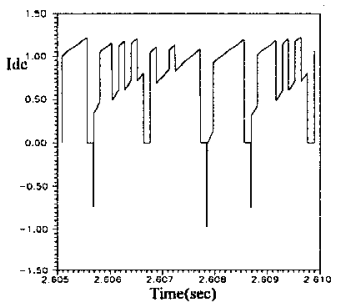

(c)

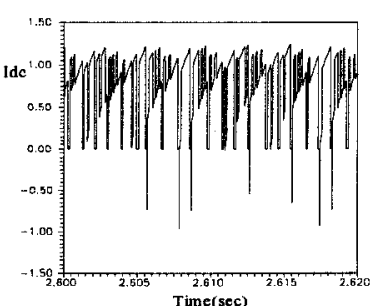

(b)

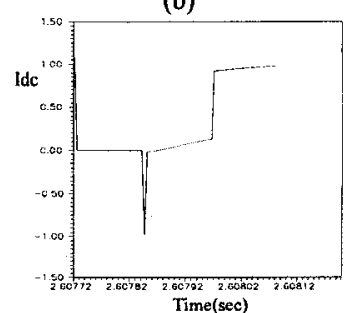

(d)
Fig. 8. Negative spikes at DC link current. (a) Dead time is not considered (20 ms). (b) Dead time is considered $(20 \mathrm{~ms})$. (c) Dead time is considered (zoomed to $5 \mathrm{~ms}$ ). (d) Dead time is considered (zoomed to one subcycle).

\section{B. Experimental Verification}

The experimental waveform of DC link current for one fundamental period $(20 \mathrm{~ms})$ is shown in Fig. 9. The agreement between the simulation and measurement results verifies the correctness of the simulation.

Fig. 10 shows the measured waveforms of DC link current and its corresponding inverter upper leg current. It can be observed that there are both negative spike and positive spikes at the measured DC link current waveform while there is only positive spike on the measured leg current, which is mainly caused by the reverse recovery effect of diode. Moreover, the measured negative spike shows that its duration closely agrees with the dead time interval of $10 \mu \mathrm{s}$ and it happens at the beginning of this subcycle, which is usually dead time interval. When the duration of dead time is changed, which is software-controlled inside of the inverter, the duration of the negative spike is changed correspondingly also. Furthermore not in every subcycle there exists this kind of negative spike (Fig. 11). Thus, it can be verified that the negative spike is caused by dead time and 
influenced by the system operation. It should be pointed out that since the negative spike is with very short time duration and there is no regularity in practical system, the oscilloscope should be worked at trigger mode to capture the negative spikes. And the idealization of switching devices in theory analysis also causes a little difference on the shapes of current spikes between simulation and experimental ones.

\section{CONCLUSION}

With the introduction of novel concept DTV, DC link current of PWM inverter drives has been investigated deeply and comprehensively. Unified expressions for DC link current are derived and some new knowledge on DC link current are acquired for both steady and transient operations. And the phenomenon of negative spikes caused by dead time on DC link current has been confirmed by means of theoretical analysis, computer simulation and experimental results. Hence the information of dead time can be easily acquired from the DC link current. DTV has been verified as a useful tool for inverter analysis. The further utilization of DTV, such as system stability analysis and compensation of dead time effect, will be investigated.

\section{APPENDIX}

Parameters of the induction motor (per unit):

inertial constant $H=300$, stator resistance $R_{s}=0.03002$, rotor resistance $R_{r}=0.02686$, stator reactance $L_{s}=3.6775$, rotor reactance $L_{r}=3.6775$, mutual reactance $L_{m}=3.5775$.

\section{REFERENCES}

[1] P. D. Evans and R. J. Hill-Cottingham, "DC link current in PWM inverters," IEE Proc. - B, vol. 133, pp. 217-224, 1986.

[2] T. C. Green and B. W. Williams, "Derivation of motor line-current waveforms from the DC-link current of an inverter," IEE Proc. - B, vol. 136, pp. 196-204, 1989.

[3] R. S. Colby and D. W. Novotny, "An efficiency optimizing permanent magnet synchronous motor drive," IEEE Trans. Ind. Appl., vol. 24, pp. 462-469, 1988.

[4] T. G. Habetler and F. Profumo, "Direct torque control of induction machines using space vector modulation," IEEE IAS Ann. Meet. 1991, pp. 428-436.

[5] S. G. Jeong and M. H. Park, "The analysis and compensation of dead time effects in PWM inverters," IEEE Trans. Ind. Electron., vol. 38, pp. $108-114,1991$.

[6] J. W. Choi and S. K. Sul, "Inverter output voltage systhesis using novel dead time compensation," IEEE Trans. Pow. Electron., vol. 11, pp. 221-227, 1996.

[7] Y. Li, L. Huang and F. Li, "All digital space vector PWM induction motor drive based on flux control," Proc., IEEE TENCON, 1993, vol. 5, pp. 598-601.

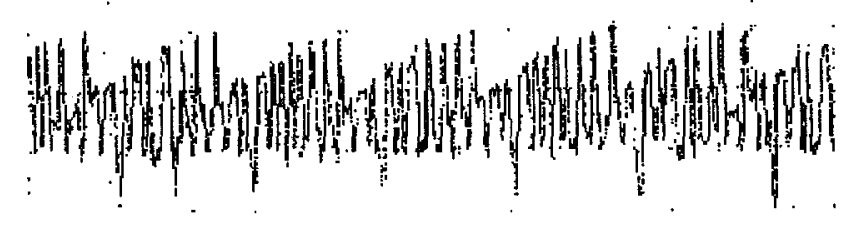

Fig. 9. Measured waveform of DC link current over $20 \mathrm{~ms}$.

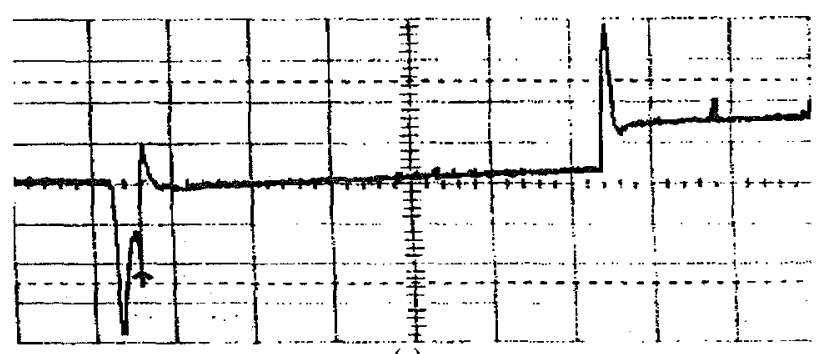

(a)

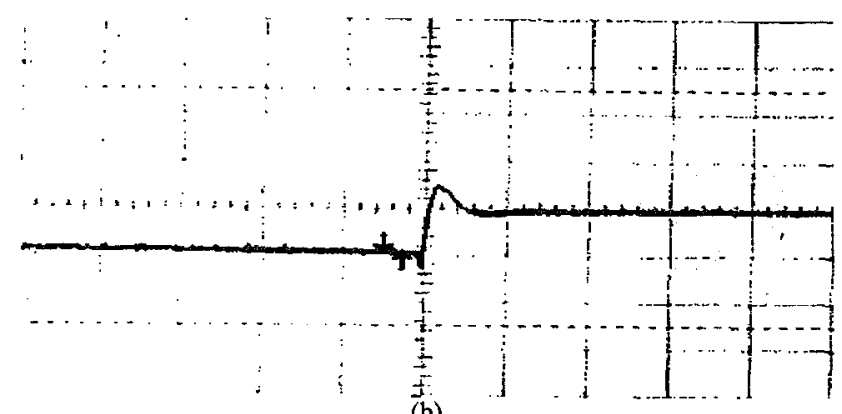

(b)

Fig 10. Measured current waveforms with occurrence of negative spikes (a) DC link current $(20 \mu \mathrm{s} / \mathrm{div})$. (b) Upper leg current $(10 \mu \mathrm{s} / \mathrm{div})$.

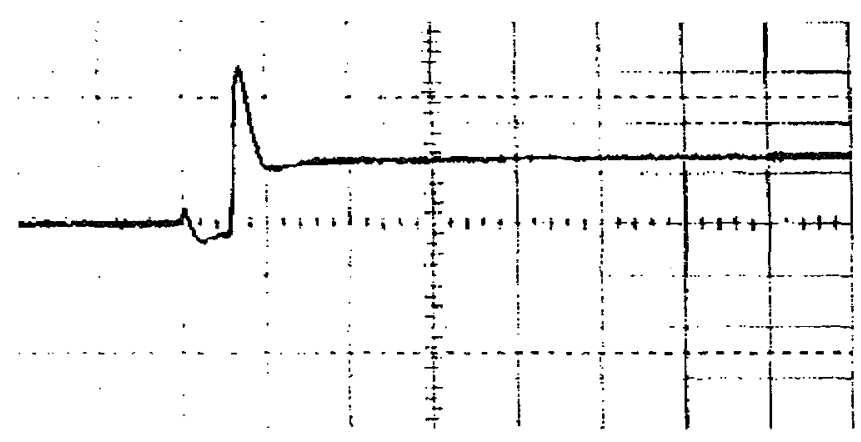

Fig. 11. Measured DC link current waveform without occurrence of negative spikes $(10 \mu \mathrm{s} / \mathrm{div})$. 